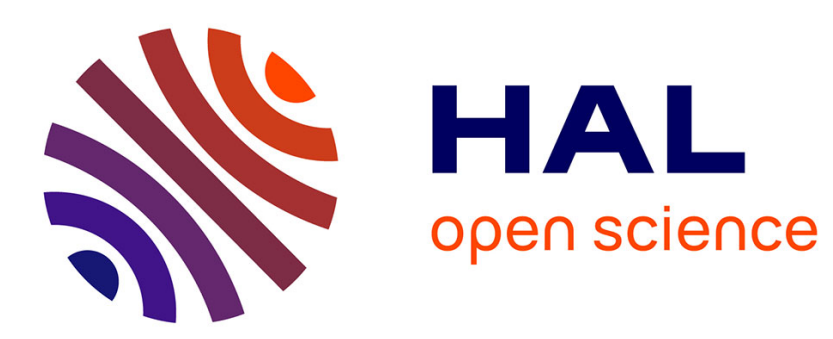

\title{
On the transfer of cascades from primary damage codes to rate equation cluster dynamics and its relation to experiments
}

\author{
T. Jourdan, J.-P. Crocombette
}

\section{- To cite this version:}

T. Jourdan, J.-P. Crocombette. On the transfer of cascades from primary damage codes to rate equation cluster dynamics and its relation to experiments. Computational Materials Science, 2018, 145, pp.235-243. 10.1016/j.commatsci.2018.01.009 . cea-02428745

\section{HAL Id: cea-02428745 \\ https://hal-cea.archives-ouvertes.fr/cea-02428745}

Submitted on 6 Jan 2020

HAL is a multi-disciplinary open access archive for the deposit and dissemination of scientific research documents, whether they are published or not. The documents may come from teaching and research institutions in France or abroad, or from public or private research centers.
L'archive ouverte pluridisciplinaire HAL, est destinée au dépôt et à la diffusion de documents scientifiques de niveau recherche, publiés ou non, émanant des établissements d'enseignement et de recherche français ou étrangers, des laboratoires publics ou privés. 


\title{
On the transfer of cascades from primary damage codes to rate equation cluster dynamics and its relation to experiments
}

\author{
T. Jourdan*, J.-P. Crocombette \\ DEN-Service de Recherches de Métallurgie Physique, CEA, Université Paris-Saclay, F-91191 Gif-sur-Yvette, France
}

\section{A R T I C L E I N F O}

\section{Article history:}

Received 8 September 2017

Received in revised form 29 November 2017

Accepted 3 January 2018

Available online 9 January 2018

\section{Keywords:}

Cluster dynamics

Cascades

Annealing

Monte Carlo

\begin{abstract}
A B S T R A C T
Transferring displacement cascades from primary damage codes to rate equation cluster dynamics (RECD) is not straightforward, due to the inability of RECD to treat spatial correlations explicitly. A method, called "sphere homogenization kinetic Monte Carlo" (SHKMC), has been proposed to produce a effective source term from a cascade database. This paper reviews the method and a few applications. SHKMC is based on a modified kinetic Monte Carlo algorithm to keep track of the homogenization process of defects within cascades. The crucial parameter is the homogenization distance, which is not an intrinsic parameter of cascades but which is given by RECD simulations. SHKMC leads to a time-varying source term, even under constant irradiation flux. RECD with such a source term is able to reproduce reference kinetic Monte Carlo calculations of microstructure evolution under cascade conditions. It is also possible to provide a spatially-dependent source term for the simulation of ion irradiations. As an example, irradiation of iron with $10 \mathrm{MeV}$ Fe ions is discussed. Analysis of the source term shows that the fraction of mono-defects is close to the fraction of freely-migrating defects determined experimentally and that it significantly varies with depth.
\end{abstract}

(c) 2018 Elsevier B.V. All rights reserved.

\section{Introduction}

The simulation of nucleation and growth of interstitial and vacancy clusters under irradiation can be performed with various methods, such as kinetic Monte Carlo (KMC) [1-4], phase field [5] and rate equation cluster dynamics (RECD) [6-11]. Among these methods, RECD is in general the most efficient owing to its mean field formalism. Ordinary differential equations on cluster concentrations are solved as a function of time. The combination of efficient time solvers [12] and numerical approximations such as grouping methods [6] or the Fokker-Planck equation [13] make this method unrivalled to reach doses as high as $100 \mathrm{dpa}$ (displacements per atom) in a few minutes of wall-clock time $[14,10,15]$. For systems with clusters containing three types of elements or more, deterministic solving becomes numerically difficult and stochastic approaches can be used $[16,17,11]$.

Efficiency of the RECD approach is obtained at the cost of substantial efforts to determine the parameters of the model. Among other things, cluster free energies [18-20] and reaction rates between clusters (so-called sink strengths) $[21,22]$ are quantities

\footnotetext{
is This article belongs to the special issue: Radiation Effects.

* Corresponding author.

E-mail address: thomas.jourdan@cea.fr (T. Jourdan).
}

that can be calculated from atomistic simulations. It has been shown that once it is properly parametrized, RECD gives results in good agreement with reference atomic KMC calculations under thermal aging $[18,19,23]$. Under irradiation, when isolated defects are produced, RECD results are also consistent with simulations by object kinetic Monte Carlo (OKMC) [24-26] or event kinetic Monte Carlo (EKMC) [27]. Under ion and neutron irradiations, an additional difficulty arises. Defects are produced inside displacement cascades, where their positions are spatially correlated. Spatial correlations cannot be taken into account directly in the formalism of RECD: due to its fundamental mean field hypothesis, it is assumed that defects are homogeneously distributed in the material. Therefore, simulations of cascade damage annealing $[28,29]$ and irradiation [25] lead to marked differences between RECD and O/EKMC calculations (called hereafter KMC for simplicity).

To circumvent this problem, several methods have been proposed. All of them need a cascade database produced by a primary damage code, such as molecular dynamics (MD) or binary collision approximation (BCA) codes. The simplest method, based on the annealing of cascades, is also the oldest one. Originally it was motivated by the determination of the fraction of freely migrating defects (FMDs), ie defects which escape the cascade region and can diffuse over long distances to contribute, for example, to void and loop growth [30-32]. Then it was used to justify the concept of "production bias" in theories of void swelling [33,34]. It seems 
that it became popular to provide source terms for RECD only recently [35,36]. It consists in annealing each cascade with KMC (MD cannot simulate such large times), counting the remaining clusters after annealing and averaging the number of clusters of each type over the KMC runs to provide effective quantities of clusters created in cascades. Such quantities serve as effective source terms in RECD. During annealing, defects diffuse in the matrix, so they are more randomly distributed at the end of the simulation. It can be expected that if the annealing is sufficiently long, defects are nearly homogeneously distributed and the transfer to RECD is valid.

However, it is not possible to define a proper annealing time in all cases. It is quite common that cluster diffusion coefficients span several orders of magnitude. As an example, in $\alpha$-iron, taking vacancy and interstitial migration energies equal to $E_{v}^{\mathrm{m}}=0.67 \mathrm{eV}$ and $E_{i}^{\mathrm{m}}=0.34 \mathrm{eV}$ respectively and the same diffusion prefactor, at $300 \mathrm{~K}$ the ratio of interstitial to vacancy diffusion coefficients is around $3.5 \times 10^{5}$. Therefore, in this case, interstitials will homogenize much more rapidly than vacancies. If the annealing time is limited to the point when interstitials can be considered homogeneously distributed, vacancies will have hardly diffused and they will still be spatially correlated. Thus the transfer to RECD is not expected to be completely satisfactory. Incidentally, another difficulty in the annealing method is to estimate when a defect can be considered as homogenized. As a first approximation, it is the case when the defect reaches the boundaries of the KMC simulation box, if the box dimension $l$ is around $l=d^{-1 / 3}$, where $d$ is the density of cascades [35]. Since this density varies with time, it appears difficult to find the correct value for $l$.

The second method, which is the topic of this article, is called the "sphere homogenization" KMC (SHKMC) method [37]. It was specifically designed to alleviate the problems inherent to the annealing method. It also relies on the annealing of defects, in the sense that defects are allowed to diffuse in a KMC box. However, a precise diffusion distance is given to estimate when defects are considered as homogenized. Such defects are handled in a specific way, to avoid them to perturb slower defects which are still not homogenized. The choice of the simulation time appears much less critical than in the annealing method; in general, it should be high enough for the slowest defects to homogenize, regardless of the fastest species. Using this method, it was shown that RECD simulations are in very good agreement with KMC simulations for irradiations with $20 \mathrm{keV}$ primary knock-on atoms (PKAs) and $60 \mathrm{keV}$ helium ions in $\alpha$-iron.

Finally, a third method was recently proposed to incorporate cascade damage in RECD $[38,11]$. It is somewhat different from the two previously discussed methods, since it relies on a specific version of cluster dynamics, called "spatially resolved stochastic cluster dynamics" (SRSCD). In this kind of model, a system is divided into cells where classical mean field RECD equations are solved. Cells are coupled together by the diffusion of mobile clusters. This method bears some resemblance to spatial 1Ddiscretization which is performed in RECD to simulate depthdependent microstructures [39,9,10], but here the discretization is performed along the three spatial dimensions. Due to the large number of equations to solve, a stochastic approach, similar to the one proposed in Ref. [16], is used. It was shown that provided the cell size is similar to the cascade size (around $10 \mathrm{~nm}$ for $20 \mathrm{keV}$ PKAs), cascade effects can be simulated by directly injecting the number of defects due to a cascade into the appropriate cells.

In this article we review the SHKMC method presented in Ref. [37]. Simulation techniques are briefly reminded in Section 2. The SHKMC method is described in Section 3 and a few applications in $\alpha$-iron are shown and discussed in Section 4. In Section 4.1 the validity of the method is assessed for the irradiation with 20
$\mathrm{keV}$ PKAs. The sensitivity of RECD results to the number of cascades in the cascade database is discussed in Section 4.2. Finally, SHKMC is used as a standalone technique to provide an estimation of the FMD fraction and to investigate its variation with depth for a heavy ion irradiation (Section 4.3).

\section{Simulation techniques}

Our goal is to simulate cluster distributions as a function of time under cascade damage conditions. For this purpose we first need a cascade database, produced by a primary damage code. A reference cluster distribution can then be obtained by performing a KMC simulation where cascades are introduced sequentially up to the targeted physical time. RECD calculations depend on a source term, which can be extracted directly from the cascade database (noannealing case), or after using annealing or SHKMC codes on this database. Annealing and SHKMC were in fact performed with the same KMC code as the one used for the reference calculation, where a dedicated module for damage annealing and homogenization was introduced. In this section the three different codes are briefly described and the parameters are listed. The agreement of RECD with KMC is discussed in the case where isolated defects are produced, to show the consistency of KMC and RECD formalisms.

\subsection{Primary damage calculations (iradina)}

Cascades were produced with the iradina code [40]. Iradina uses a Monte Carlo approach to compute the transport of ions in a target and the associated damage. As SRIM [41] and MARLOWE [42] codes, it relies on the BCA approximation, which means that collision cascades are approximated by a sequence of binary collisions. The computed damage is less precise than with MD: among other things, shock waves [43], thermal spike [44] and the resulting intracascade clustering are not simulated, so only monointerstitials and mono-vacancies are predicted by BCA codes. Point defect clusters are produced only by the diffusion and clustering of mono-interstitials and mono-vacancies. Source terms produced with iradina will therefore be different from source terms produced with MD and in order to compare simulation results with experiments, MD should be preferred. However, our goal here is more to emphasize methodological aspects than to discuss physical results. In the following, iradina could be replaced with MD without any difficulty.

The choice of iradina is explained by several reasons. We want a code which produces a large number of cascades in a limited amount of time, notably to investigate the sensitivity of RECD calculations to the number of cascades in the database (Section 4.2). This cannot be achieved easily with MD, so BCA codes are the natural candidates. SRIM is probably the most popular BCA code in the radiation damage community, but unfortunately the position of interstitials are not provided in output. Iradina has been shown to give results in excellent agreement with SRIM in the so-called "Full Cascade" mode, with a much higher efficiency [40]. In addition, as it is open-source, we modified it slightly to provide in output the position of interstitials and vacancies. We also added the possibility to introduce the incoming ion in the middle of the simulation box, in order to simulate the damage created by PKAs in a bulk material (Section 4.1).

In all calculations, the displacement threshold energy was $E_{\mathrm{d}}=40 \mathrm{eV}$, which is the recommended value for iron [45]. Interstitials and vacancies which are closer to each other than $r_{i v}=3.3 a$, where $a$ is the lattice parameter, are assumed to spontaneously recombine [46], so they were removed from the cascade description before the cascade database was transferred to KMC. The 
damage (in dpa) is however estimated by the total number of defects produced by iradina without the recombinations.

\subsection{Kinetic Monte Carlo (JERK)}

To produce reference kinetic simulations, we use the EKMC code JERK $[1,4]$. As in OKMC codes, clusters are considered as single objects, which can migrate in the matrix, absorb and emit defects. Besides the creation rate, input parameters for these KMC codes are cluster diffusion coefficients, reaction distances (including bias values) and binding energies. These parameters are presented in next section, in the context of RECD, and listed in Table 1.

Contrary to OKMC codes, not all atomic jumps are simulated in EKMC. Instead, the total physical time is subdivided into timesteps $\Delta t$ and events (cascade, absorption or emission of a migrating defect by a cluster) that can occur during this time interval are performed; at the end of each $\Delta t$, defects are moved with single macro-jumps according to the continuous diffusion law. Of course, if $\Delta t$ is reduced down to the typical time for an atomic jump, the number of absorption events during $\Delta t$ decreases and at the end of $\Delta t$, defects will undergo atomic-like jumps, so EKMC becomes equivalent to OKMC. It has been shown that EKMC and OKMC lead to results in very good agreement, but that EKMC is typically faster than OKMC [4], provided that the list of possible events during $\Delta t$ is built rapidly. For this purpose, a modification of the original algorithm [4] has been introduced [37]. The simulation box is divided into rectangular cells which contain at most a specified number of defects (typically 100). Speed-up is obtained by limiting the calculation of absorption events of a given defect to defects in the same cell and in the neighbour cells. For this calculation to be valid, $\Delta t$ must be sufficiently low so the probability that a defect interacts with defects located beyond the neighbour cells is negligible. In practice, $\Delta t$ is chosen such that $2 D_{\max } \Delta t=\left(\Delta x_{\min }-d_{\max }\right)^{2} / 4$, with $D_{\max }$ the diffusion coefficient of the fastest defect in the simulation box, $d_{\max }$ the largest reaction distance between defects and $\Delta x_{\min }$ is the smallest cell dimension. There are two advantages to use this method. The first one is that the calculation in JERK now scales linearly with the number of clusters instead of quadratically as with the original algorithm. In addition, the timestep depends on the clusters present in the simulation box: if interstitials disappear and only vacancies remain, $D_{\max }$ decreases by several orders of magnitude and the timestep increases in the same proportion. These two improvements lead to considerable speed-up in practice, especially for the homogenization of large cascades (see Section 4.3).

\subsection{Rate equation cluster dynamics (CRESCENDO)}

As KMC methods, RECD considers the microstructure as a cluster gas: clusters can migrate by random walk in the matrix, capture and emit defects. Input parameters of RECD are essentially the same as for KMC. The main difference is that the positions of clusters are not considered, only their concentration is retained. Equations of the following type are numerically solved in the RECD code CRESCENDO [10]:

$\frac{\mathrm{d} C_{n}}{\mathrm{~d} t}=G_{n}+\sum_{m \in \mathcal{M}} J_{n-m, m}-\sum_{m \in \mathcal{M}} J_{n, n+m}-\sum_{m \in \Omega} J_{m, n+m}$,

where $\Omega$ is the set of all clusters, $\mathcal{M}$ is the restriction of $\Omega$ to mobile clusters, $C_{n}$ is the concentration of clusters containing $n$ defects, $G_{n}$ is the creation rate of cluster type $n$ and $J_{n, n+m}$ is a net reactive flux between classes $n$ and $n+m$, due to the mobility of cluster $m$ only (even if $n$ is mobile). The reason why the mobility of $n$ is excluded is that it is accounted for in the last term of Eq. (1). The flux reads

$J_{n, n+m}=\beta_{n, m} C_{n} C_{m}-\alpha_{n+m, m} C_{n+m}$

In this equation, $\beta_{n, m}$ is the absorption rate of $m$ by $n$, which is usually determined by solving the stationary diffusion equation around the cluster. For three-dimensional diffusion of defects, in the dilute limit, which is the case considered here, it can be written as

$\beta_{n, m}=4 \pi d_{n, m} D_{m}$

where $d_{n, m}$ is a reaction distance and $D_{m}$ is the diffusion coefficient of cluster type $m\left(D_{m}=D_{0, m} \exp \left(-E_{m}^{\mathrm{m}} / k T\right)\right.$, with $D_{0, m}$ the diffusion prefactor and $E_{m}^{\mathrm{m}}$ the migration energy). Reaction distances for loops and cavities are taken from [47] and are an extension of those used in Ref. [7].

The emission coefficient is given by

$\alpha_{n+m, m}=\frac{\beta_{n, m}}{V_{\mathrm{at}}} \exp \left(-\frac{F_{n+m, m}^{\mathrm{b}}}{k T}\right)$,

where $F_{n+m, m}^{\mathrm{b}}$ is the binding free energy of cluster $m$ to cluster $n$ and $V_{\text {at }}$ is the atomic volume.

In the following, since interstitial and vacancy clusters are considered, we use negative values of $n$ for vacancy clusters and positive values for interstitial clusters. Input parameters are shown in Table 1 for all calculations reported in this article, and correspond to those of Ref. [37]. More complex parametrizations, taking into account loop mobility and trapping [3] could be considered for a quantitative comparison to experiments.

Table 1

Parameters used for the simulation of iron irradiation. Migration and binding energies are from Ref. [49].

\begin{tabular}{|c|c|c|c|}
\hline Symbol & Description & Value & Unit \\
\hline \multicolumn{4}{|c|}{ BCA } \\
\hline$E_{\mathrm{d}}$ & Displacement threshold energy & 40 & $\mathrm{eV}$ \\
\hline \multicolumn{4}{|c|}{ KMC/RECD } \\
\hline$V_{\text {at }}$ & Atomic volume & 0.0118 & $\mathrm{~nm}^{3}$ \\
\hline$D_{0, n}(n \in[-4,3] \backslash\{0\})$ & Diffusion prefactor & $8.2 \times 10^{-7}$ & $\mathrm{~m}^{2} \mathrm{~s}^{-1}$ \\
\hline$E_{n}^{\mathrm{m}}(n=1,2,3)$ & Migration energy of interstitial clusters & $0.34,0.42,0.43$ & $\mathrm{eV}$ \\
\hline$E_{n}^{\mathrm{m}}(n=-1,-2,-3,-4)$ & Migration energy of vacancy clusters & $0.67,0.62,0.35,0.48$ & $\mathrm{eV}$ \\
\hline$F_{n, 1}^{\mathrm{b}}(n=2,3,4)(n \geqslant 5)$ & Binding energy of interstitials to int. clusters & $0.8,0.92,1.64$ & $\mathrm{eV}$ \\
\hline$F_{n,-1}^{\mathrm{b}}(n=-2,-3,-4,-5)(n \leqslant-6)$ & Binding energy of vacancies to vac. clusters & $\begin{array}{l}\text { Capillary law } \\
0.30,0.37,0.62,0.72\end{array}$ & $\mathrm{eV}$ \\
\hline n,-1 द & Temperature & $\begin{array}{l}\text { Capillary law } \\
300\end{array}$ & K \\
\hline \multicolumn{4}{|c|}{$\mathrm{BCA} / \mathrm{KMC} / \mathrm{RECD}$} \\
\hline$r_{i v}$ & Recombination distance & 0.947 & $\mathrm{~nm}$ \\
\hline
\end{tabular}




\subsection{On the agreement between KMC and RECD}

It has been shown previously that KMC and RECD lead to very similar results when defects are produced randomly in the simulation box $[24,25]$. Here we briefly report on the agreement that can be expected between KMC and RECD approaches for our simulation conditions. To that end we compare cluster distributions when single point defects are created randomly in the simulation box. To make subsequent comparison with cascade simulations relevant, $20 \mathrm{keV}$ cascades were produced with iradina and the number of defects after recombinations was used to determine the creation rate. The dpa rate, however, is calculated with the number of defects before recombinations. We chose here a dose rate of $10^{-4} \mathrm{dpa} \mathrm{s}^{-1}$ and a final dose of $10^{-3} \mathrm{dpa}$. The temperature is fixed to $300 \mathrm{~K}$. A total number of $1000 \mathrm{KMC}$ simulations were performed with simulation box size of $(400 a)^{3}$, in order to get a precise description of cluster distributions.

Cluster distributions are shown in Fig. 1. A good agreement is obtained for both interstitial and vacancy clusters. In particular, the position and the magnitude of the concentration peak for interstitial clusters is well reproduced by RECD calculations. Some differences are observed for small vacancy clusters. Besides the statistical errors, these differences can be due to the low binding energy of small vacancy clusters. Even at $300 \mathrm{~K}$, the probability of dissociation is not completely negligible. It has been shown that obtaining consistency between RECD and KMC for emission is far more difficult than for absorption [48].

It is interesting to note the difference in wall-clock time to perform the two calculations: altogether the $1000 \mathrm{KMC}$ runs required around 80 days, while the cluster dynamics calculation, which required only one run due to its deterministic nature, lasted only $11 \mathrm{~s}$. Therefore we see how crucial it is to have a method to model not only isolated but also cascade damage in RECD.

\section{Transferring primary damage to RECD: the SHKMC method}

The SHKMC method relies on a KMC code to provide an effective source term for RECD. As we will see, it only requires a minor modification of the KMC code and can be applied to both EKMC and
OKMC. Once a cascade database has been produced, cascades are homogenized one by one, separately, in a SHKMC run. Input parameters of the homogenization procedure are a distance $r_{\mathrm{h}}$, called the homogenization distance, and the simulation time $t_{\mathrm{f}}$. They will be discussed hereafter. For each cascade, the following steps are performed:

1. Insert the cascade in the KMC simulation box.

2. Create spheres of radius $r_{\mathrm{h}}$ around each cluster. The position of these spheres remains fixed throughout the simulation.

3. Perform the kinetic simulation. Once a cluster crosses its sphere, it is considered as homogenized. It is removed from the simulation and added to the source term for RECD. The simulation proceeds with the remaining clusters until the final time $t_{\mathrm{f}}$ is reached.

4. At $t=t_{\mathrm{f}}$, add the remaining clusters to the source term for RECD.

The final RECD source term is the average of all source terms produced with the different homogenizations of cascades. The crucial parameter in the SHKMC method is the homogenization distance $r_{\mathrm{h}}$. This distance must be somehow related to the distance between defects in RECD calculations; it is not a parameter determined from the cascade database. For example, two clusters a few nanometers apart may be considered as either correlated if the overall density of clusters in the material is low (for example, at the beginning of irradiation), or uncorrelated if all defects are a few nanometers away (it is the case at high irradiation dose, especially at low temperature).

Therefore, we choose to define the homogenization distance from RECD as follows:

$r_{\mathrm{h}}=\frac{1}{2}\left(\frac{1}{\sum_{n \in \Omega} C_{n}}\right)^{1 / 3}$.

The simulation time, on the contrary, is a less constrained parameter. In general, it should be large enough for all defects to homogenize during the SHKMC run. It is a criterion that may be hard to fulfill at low temperature, where some defects are nearly immobile over the experimental timescale. In this case, it seems

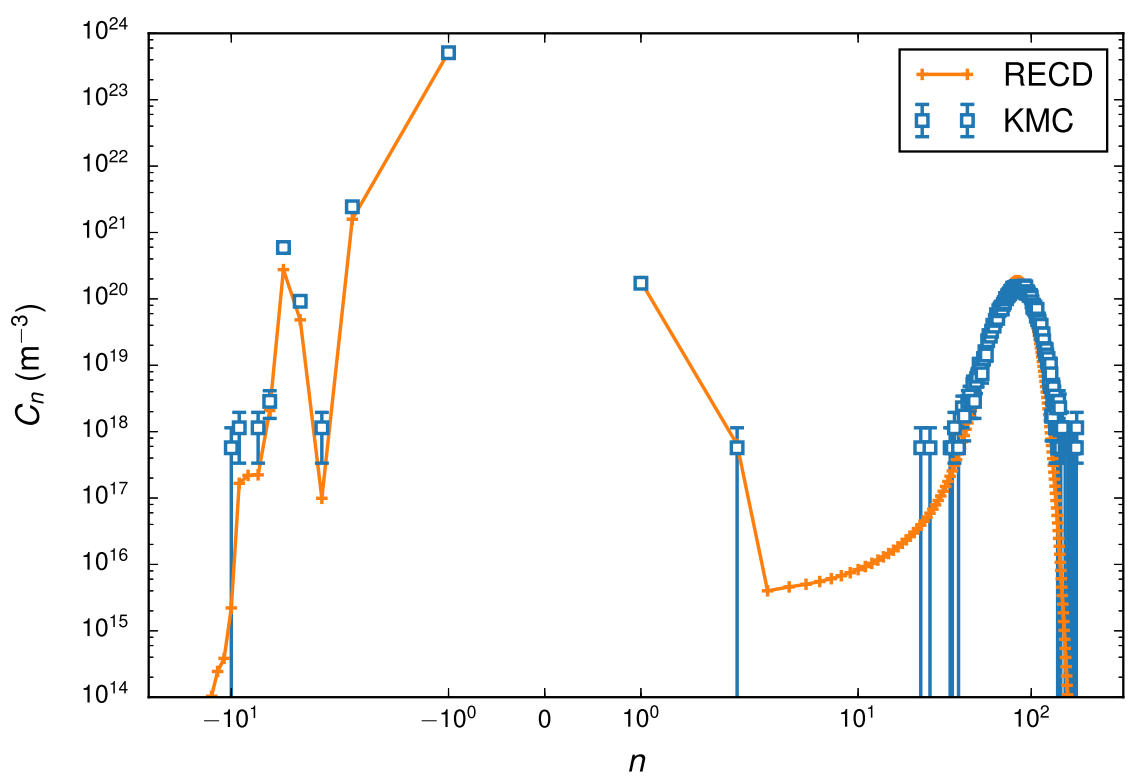

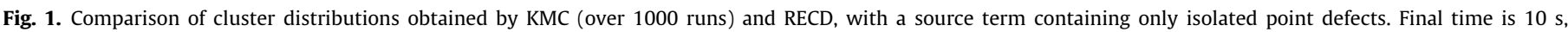

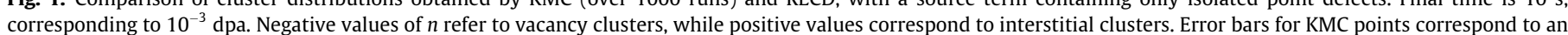

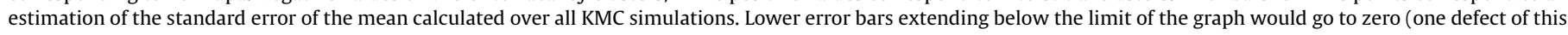
type is observed over all KMC simulations). 


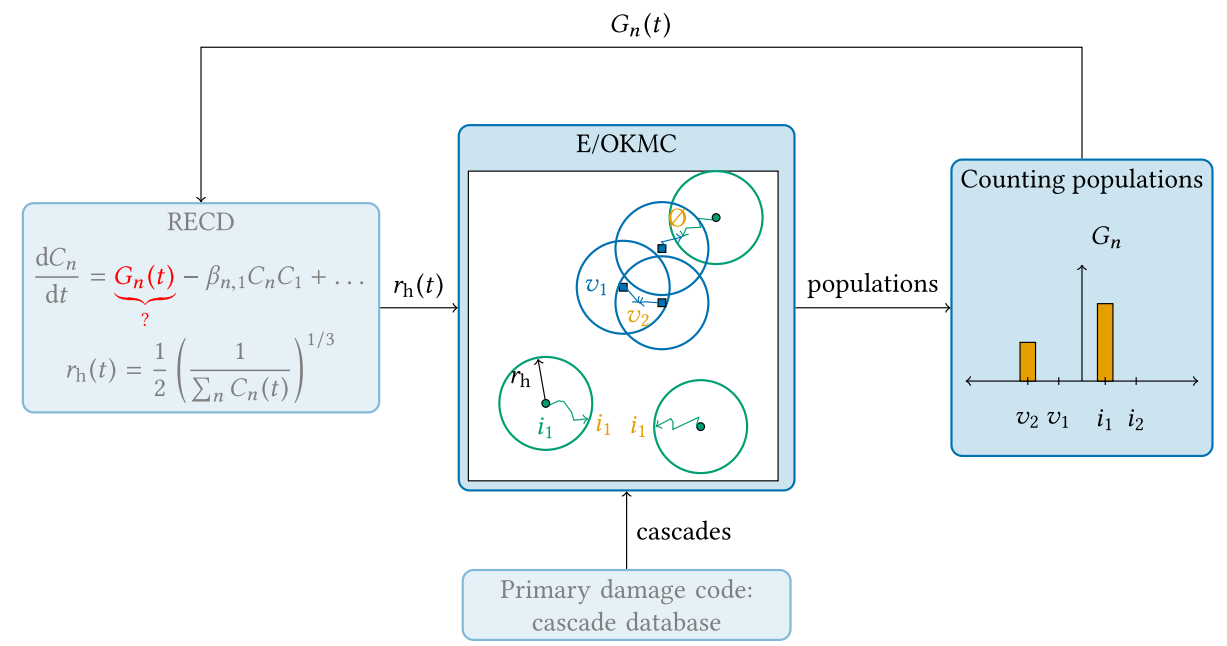

Fig. 2. Schematic illustration of the SHKMC method.

impossible to design a proper effective source term by this method, as clusters will not be homogenized enough whatever the value of $t_{\mathrm{f}}$. Another constraint is that $t_{\mathrm{f}}$ should be lower than the final time of the physical simulation. If it is not the case, events which are too slow to occur during the physical simulation could be wrongly taken into account in the effective source term.

Since concentrations $C_{n}$ evolve with time, it is also the case of $r_{\mathrm{h}}$. Therefore homogenization of the cascade database should be performed at every timestep of a RECD calculation with the value of $r_{\mathrm{h}}$ deduced from the concentrations, to provide the appropriate effective source term for the next timestep (Fig. 2). In practice, it is more convenient and efficient to prepare a set of source terms obtained with different values of $r_{\mathrm{h}}$ and to interpolate the source term at every timestep. It is important to note that the SHKMC method leads to a varying source term, even if the source term used in the reference KMC run stays constant. The relevance of the varying source term is seen at sufficiently large doses (Section 4.1).

Finally, storage of the source term in steps 3 and 4 of the SHKMC method can be done within predefined slices of the SHKMC box, in order to provide a spatially-resolved source term. This is especially useful for the simulation of ion irradiations. The method is described and discussed in Ref. [37]. An example is shown in Section 4.3.

\section{Applications of the SHKMC method}

\subsection{Irradiation with $20 \mathrm{keV}$ PKAs in $\alpha$-iron}

Bulk irradiation of $\alpha$-iron with $20 \mathrm{keV}$ PKAs at $300 \mathrm{~K}$ was simulated up to $10^{-2} \mathrm{dpa}$, at a dose rate of $10^{-4} \mathrm{dpa} \mathrm{s}^{-1}$. These rather demanding conditions are used to test the validity of the SHKMC method. With such a high dose rate, typical of ion irradiations, the average time between two cascades is much shorter than under reactor conditions. In addition, at $300 \mathrm{~K}$, vacancies have a low mobility, so their homogenization requires a time which is of the order of a second, depending on the precise value of $r_{\mathrm{h}}$. Accordingly, the homogenization time was fixed to $t_{\mathrm{f}}=0.1 \mathrm{~s}$, so that it remains lower that the final time envisaged for the physical calculations $(t=100 \mathrm{~s})$. The final dose $\left(10^{-2} \mathrm{dpa}\right)$ was chosen sufficiently low to make KMC calculations tractable and to avoid effects of cascade overlap [50], which are not handled with SHKMC.

A cascade database containing 10,000 cascades was generated with iradina. These cascades contain in average 277 vacancies (interstitials) before recombination and 60 vacancies (interstitials) after recombination. The insertion rate of cascades in reference KMC simulations is fixed to $5.78 \mathrm{~s}^{-1}$, so that in boxes of size $(200 a)^{2}$, the damage rate is exactly $10^{-4} \mathrm{dpa} \mathrm{s}^{-1}$. A reference cluster distribution at $10^{-2}$ dpa was obtained by averaging cluster distributions over $1000 \mathrm{KMC}$ runs.

Different RECD source terms were produced from this database. They are shown in Figs. 3 and 4, together with the associated cluster distribution simulated by RECD. The reference KMC cluster distribution is also shown for comparison. The simplest source term (Fig. 3(a)) contains only mono-vacancies and mono-interstitials. As expected, the agreement with KMC is very poor. In particular, a few large interstitial clusters are obtained with RECD, whereas a large number of small clusters are present in KMC simulations. This is due to the fact that RECD does not handle intra-cascade clustering and therefore underestimates cluster nucleation rate if only monomers are present in the source term.

This problem can be partially solved by annealing the cascade database. In Fig. 3(b-d) the source terms and cluster distributions are shown for three annealing times $t=0, t=10^{-4}$ and $10^{-1} \mathrm{~s}$, using periodic boundary conditions in a KMC box of size $(400 a)^{3}$. We will call this method the "constant annealing time method" (CAT). The goal of the zero time annealing is just to trigger agglomerations which occur without diffusion, due to the fact that defects are closer than their reaction distance. This is typically the kind of reactions which are handled during MD simulations. We see that clusters are now present in the source term. Although the annealing time is zero, large vacancy clusters are created due their proximity in the cascade core. The agreement is better than with the previous source term, but significant differences still appear. The same kind of conclusion should hold for a cascade database produced by MD. Therefore, it appears that transferring damage directly from MD to RECD, without any further annealing or homogenization, is inaccurate.

The second annealing time $t=10^{-4} \mathrm{~s}$ has been chosen because it corresponds to the typical time for mono-interstitials to reach the box's boundaries. We can therefore estimate that at $t=10^{-4} \mathrm{~s}$, interstitials are homogenized. Contrary to the case $t=10^{-11} \mathrm{~s}$, cluster nucleation is now slightly overestimated. This overestimation is due to the fact that in the annealing simulation, interstitials can react with all other interstitials which belong to the same cascade. The effect is even more pronounced for an annealing up to $10^{-1} \mathrm{~s}$. The problem can be solved by decreasing the annealing time down to around $t=10^{-5} \mathrm{~s}$ [37], but the agreement remains relatively poor 

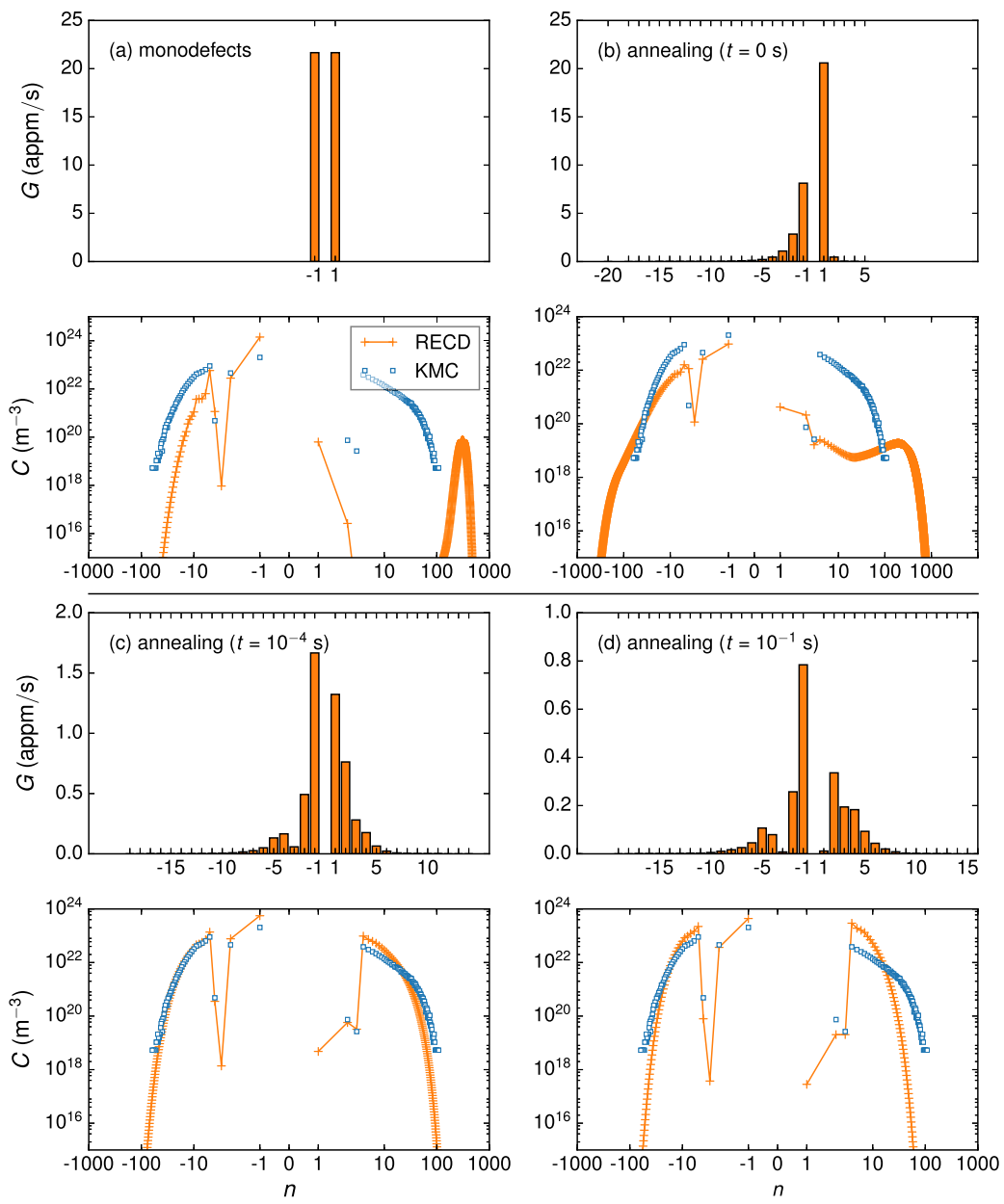

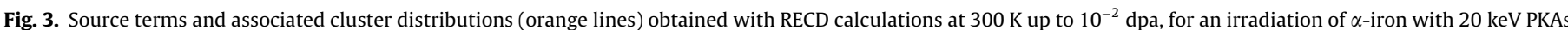

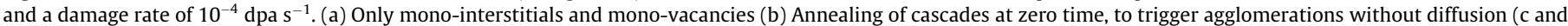

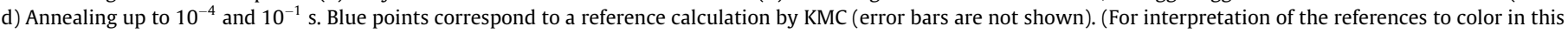
figure legend, the reader is referred to the web version of this article.)
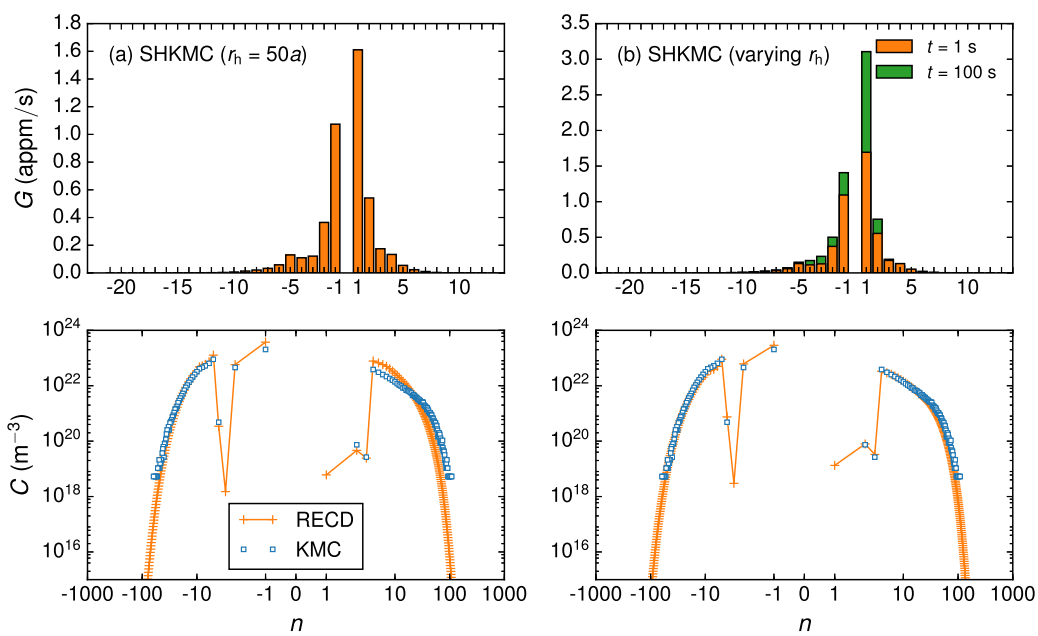

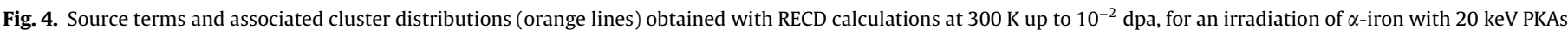

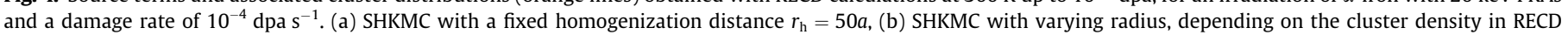

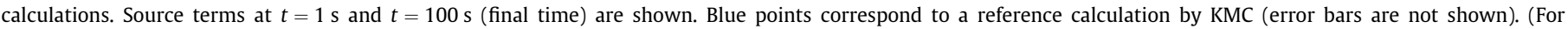
interpretation of the references to color in this figure legend, the reader is referred to the web version of this article.)

for the small mobile vacancy clusters whose concentration is overestimated by RECD. Increasing the annealing time marginally improves the result for small vacancy clusters, so the problem only partially comes from vacancies being not enough homogenized.
The other reason for this discrepancy is found by comparing CAT results to the SHKMC calculation with a fixed radius $\left(r_{\mathrm{h}}=50 a\right.$, Fig. 4(a)). One of the major differences is the higher proportion of mono-interstitials created in the source term with 
the SHKMC method. During the homogenization process, some interstitials reach their spheres and are included in the source term as such, whereas in the CAT method, they can diffuse and cluster with all defects in the cascade. This decreases the concentration of interstitials in RECD for simulations with CAT source terms, so the recombination rate with vacancies is also reduced. Vacancy concentration is thus overestimated in RECD when the CAT method is used. On the contrary, the agreement on vacancy concentration is better with the SHKMC method, while keeping the same agreement on the interstitial side.

If the variation of the source term with time is taken into account in SHKMC, RECD and reference KMC simulations are in nearly perfect agreement (Fig. 4(b)). As time increases, cluster density increases in RECD, so $r_{\mathrm{h}}$ decreases in the homogenization process. As a consequence, the concentration of interstitials rises significantly, which lowers even more the vacancy concentration due to enhanced recombinations in RECD. In addition, clustering of interstitials in SHKMC is less pronounced than when $r_{\mathrm{h}}$ is fixed to $50 a$, which decreases the nucleation rate of interstitial clusters and improves the agreement with KMC. This means that by using $r_{\mathrm{h}}=50 a$, some reactions between interstitials are performed whereas they should be treated by RECD. The additional computation cost induced by the use of a time-varying source term is relatively modest: it increases from $4.7 \mathrm{~s}$ with $r_{\mathrm{h}}=50 \mathrm{a}$ to $6.7 \mathrm{~s}$. It is not only due to a numerically more difficult time integration when the source term varies, but also to the larger cluster space needed in this particular case.

This example shows that in general, finding the optimal annealing time to provide the source term for RECD is difficult, if not impossible, owing to the large differences in cluster mobilities. SHKMC provides a simple way to homogenize all clusters, while suppressing spurious reactions that can be caused by fast defects migrating over too large distances during annealing.

\subsection{Sensitivity of RECD results to the cascade database}

We have seen in the previous section that RECD results are very sensitive to the source term values. The source term depends not only on the homogenization method, but also on the cascade database itself. In particular, the number of cascades in the database may affect the source term and the RECD results. With BCA codes, the number of cascades in the database can be as large as needed, due to the very short computation time to generate a cascade. This is not the case of cascade databases produced by MD, which rarely contain more than a few hundreds of cascades. Therefore, it appears important to estimate the impact of the number of cascades used to generate the source term on RECD results.

Starting from a cascade database for $20 \mathrm{keV}$ PKAs containing $10^{5}$ cascades, we produced 1000 source terms by homogenizing $N_{\text {casc }}$ cascades with SHKMC. Three different values of $N_{\text {casc }}$ were considered: $N_{\text {casc }}=10,100$ and 1000 . For each value of $N_{\text {casc }}$, 1000 RECD simulations were run with the different source terms and the maximum and minimum value for $C_{n}$ over the runs was determined for all $n$. The envelope curves generated in this way give an estimation of the dependency of RECD results on the number of cascades considered in the homogenization process. For the sake of simplicity, time variation of the source terms was not considered during the SHKMC process. The value $r_{\mathrm{h}}=50 a$ was chosen. The simulation conditions are those of Section 4.1. The reference solution is given by a source term produced by homogenizing $10^{4}$ cascades (see Fig. 4(a)).

Results are shown in Fig. 5. It can be seen that building a source term with only 10 cascades can lead to results which differ from the reference solution by more than one order of magnitude. Even with 100 cascades, the scattering of results is rather high, so in general it is a good practice to include several hundreds of cascades to avoid any side-effect of the cascade database on RECD results. As an example, the high dependency of long term evolution on some characteristics of primary damage calculations, such as the type of empirical potentials [51], may be reduced by just incorporating more cascades to the database.

\subsection{Spatial dependency of the source term: irradiation with $10 \mathrm{MeV}$ ions in $\alpha$-iron}

Under ion irradiation, damage is heterogeneous along the irradiated depth and it is maximum close to the region where ions come to rest. In such conditions, the source term for RECD is thus a function of depth. It is not clear, however, whether the source

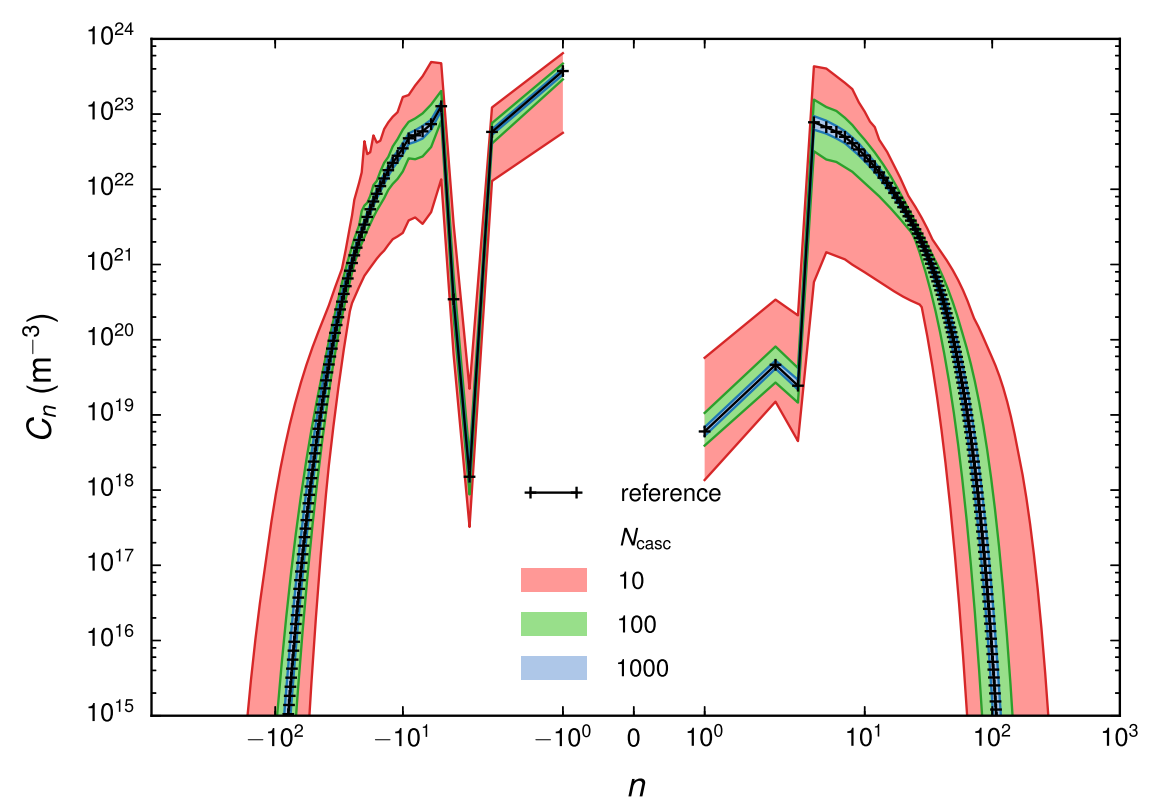

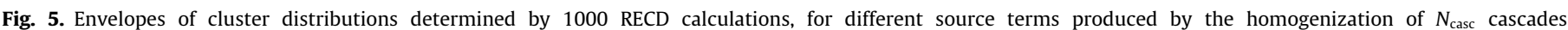

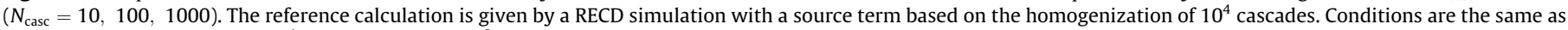
in Section 4.1: dose rate of $10^{-4} \mathrm{dpa} \mathrm{s}^{-1}$, dose of $10^{-2} \mathrm{dpa}$ and $T=300 \mathrm{~K}$. 

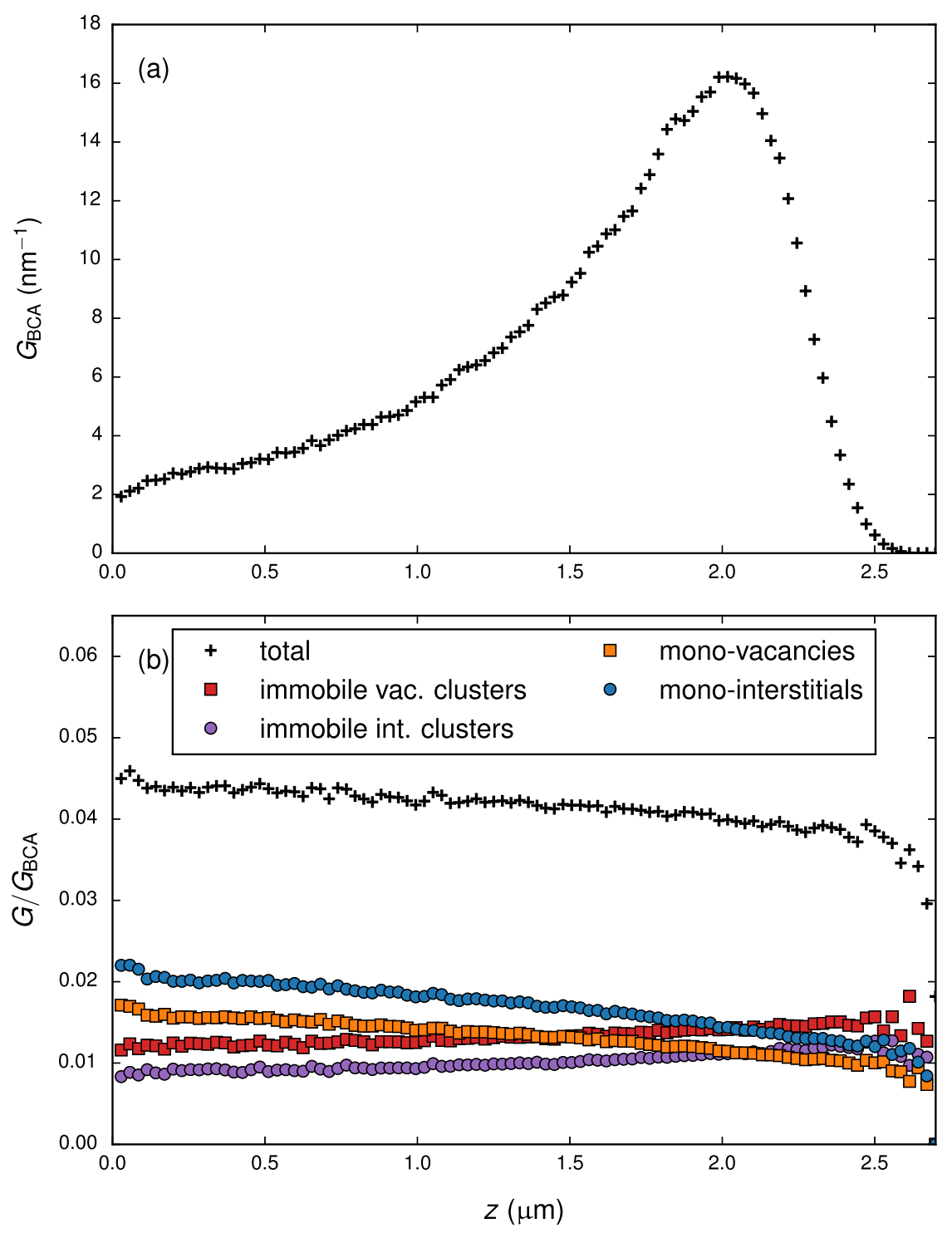

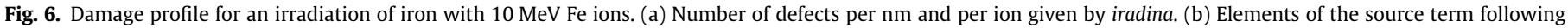
homogenization by SHKMC $\left(r_{\mathrm{h}}=50 a\right)$ : fraction of self-defects (excluding injected interstitials), vacancies and interstitials in immobile clusters and mono-defects.

terms at different depths should be proportional to each other, or if the structure of the source term should change. Since high energy PKAs lead to subcascade formation above a given threshold energy with both BCA [52,53] and MD calculations [54,55] (typically 20 $\mathrm{keV}$ in iron), as a first approximation the damage can be estimated as a combination of damage created by low energy PKAs. From this point of view, source terms at different depths computed by MD or BCA calculations following a short annealing may simply be related by a proportionality constant. As an example, the ratio of the number of defects produced by MD to the NRT value is independent of PKA energy for sufficiently high energy PKAs [55].

However, if the source term is calculated by homogenization instead of short annealing during MD or after a BCA calculation, interactions between defects from different subcascades can happen. The source term can be expected to differ near the surface and in the damage peak region, since correlations between defects are higher in the latter. Experimentally, it has been shown that the fraction of freely migrating defects (FMDs) decreases with PKA energy [56]. Although FMD fraction is not a direct measurement of the source term, it is related to the fraction of mobile clusters in the RECD source term. Therefore, a dependency of the source term on depth is plausible.
To check this hypothesis, the source term for an irradiation of $\alpha$ iron with $10 \mathrm{MeV}$ Fe ions was determined by SHKMC. Iradina was used to produce a cascade database containing 10,000 cascades. The damage profile (in number of defects produced per cascade and per unit length, $G_{\mathrm{BCA}}$ ) is shown in Fig. 6(a). This number is a direct output from iradina, without any account of recombinations. The damage significantly varies width depth and is maximum at around $2 \mu \mathrm{m}$.

Cascades were homogenized with SHKMC with a fixed homogenization distance $r_{\mathrm{h}}=50 a$. A spatially-dependent source term was calculated, using the methodology presented in Ref. [37]. To characterize the dependency of source term on depth, we represent in Fig. 6(b) the total number of vacancies and interstitials in the source term, the number of vacancies and interstitials in immobile clusters and the number of mono-defects. These quantities are normalized by $G_{\mathrm{BCA}}$. The total fraction of vacancies and interstitials slightly decreases with depth, which can be interpreted by higher spatial correlations at the end of range, so the recombination rate is larger. The decrease can be entirely ascribed to mono-defects: for example, the fraction of single interstitials is around 0.02 near the surface and only 0.01 near the damage peak. The proportion of vacancies and interstitials in clusters slightly 
increases due to stronger clustering and their fraction in mobile clusters, excluding mono-defects, stays constant (not shown).

Such a variation with depth can have a large influence on RECD results, due to the high sensitivity of RECD to the source term. A similar decrease in the production rate of mono-defects in the damage peak region was recently inferred from RECD results in austenitic stainless steels for $10 \mathrm{MeV}$ Fe ion irradiations, in order to properly model experimental microstructures [57]. In particular, it was shown that injected interstitials near the damage peak have a greater influence on void swelling if this variation is taken into account. Indeed, injected ions represent a larger part of the freely migrating mono-interstitials.

It is tempting to compare the fraction of mono-defects or mobile clusters in the source term to the experimentally measured fraction of FMDs. In our calculation, it amounts to a few percents, in agreement with the experimental values [58-60]. It should be noted, however, that BCA calculations cannot reproduce the complexity of cluster formation and that results depend on the KMC parametrization. A recent study, using MD calculations and annealings with OKMC, gives a FMD fraction of around 20\% in iron [61]. All loops were considered mobile in this study and thus could quickly escape the cascade region, which can explain why the FMD fraction is not much different from the fraction of surviving defects predicted by MD (30\%).

\section{Conclusions}

A method to transfer primary damage calculated by MD or BCA approaches to RECD has been presented. The method, called SHKMC, can be used with any KMC code with only minor modifications. It relies on a homogenization distance to estimate when clusters have diffused enough in the KMC box and should be transferred to the RECD source term. This homogenization distance is not a parameter deduced from the cascade database; it is obtained from the density of clusters in the mean field RECD calculation. This leads to a time-varying source term, which must be updated at each time step of a RECD simulation.

RECD coupled to SHKMC succeeded in reproducing reference KMC simulations of iron irradiated with $20 \mathrm{keV}$ PKAs up to $10^{-2}$ dpa. By varying the number of cascades in the homogenization process, it was shown that RECD calculations are highly sensitive to the size of the cascade database. In general, several hundreds of cascades should be included in the database to avoid any spurious effect of the source term.

SHKMC can be used to homogenize the damage produced by high energy PKAs and investigate spatial variations of the source term. As an example, the source term produced by $10 \mathrm{MeV}$ Fe ions in iron was calculated. It was shown that the fraction of monodefects varies appreciably with depth. Although the parametrization was simple and the cascades were produced by a BCA code, this fraction is in good agreement with the typical experimental values.

\section{Acknowledgments}

Part of this work was funded by the French National Research Agency (ANR) through Projects HSynThEx (ANR-10-INTB-0905) and CoIrrHeSim (ANR-11-BS09-006). The CRESCENDO code is developed in collaboration with EDF R\&D within the framework of SIMMAT project from the I3P institute.

\section{References}

[1] J.M. Lanore, Radiat. Eff. 22 (1974) 153.

[2] M.J. Caturla, N. Soneda, E. Alonso, B.D. Wirth, T. Diaz de la Rubia, J.M. Perlado, J. Nucl. Mater. 276 (2000) 13
[3] C. Domain, C.S. Becquart, L. Malerba, J. Nucl. Mater. 335 (2004) 121.

[4] J. Dalla Torre, J.-L. Bocquet, N.V. Doan, E. Adam, A. Barbu, Philos. Mag. 85 (2005) 549.

[5] A. Badillo, P. Bellon, R.S. Averback, Model. Simul. Mater. Sci. Eng. 23 (2015) 035008.

[6] S.I. Golubov, A.M. Ovcharenko, A.V. Barashev, B.N. Singh, Philos. Mag. A 81 (2001) 643

[7] A. Hardouin-Duparc, C. Moingeon, N. Smetniansky-de-Grande, A. Barbu, J. Nucl. Mater. 302 (2002) 143.

[8] C.J. Ortiz, M.J. Caturla, C.C. Fu, F. Willaime, Phys. Rev. B 75 (2007) 100102(R).

[9] D. Xu, B.D. Wirth, M. Li, M.A. Kirk, Acta Mater. 60 (2012) 4286.

[10] T. Jourdan, G. Bencteux, G. Adjanor, J. Nucl. Mater. 444 (2014) 298.

[11] A.Y. Dunn, L. Capolungo, Comp. Mater. Sci. 102 (2015) 314.

[12] A.C. Hindmarsh, P.N. Brown, K.E. Grant, S.L. Lee, R. Serban, D.E. Shumaker, C.S. Woodward, ACM Trans. Math. Softw. 31 (2005) 363, https://doi.org/10.1145/ 1089014.1089020.

[13] T. Jourdan, G. Stoltz, F. Legoll, L. Monasse, Comput. Phys. Commun. 207 (2016) 170.

[14] M. Zouari, L. Fournier, A. Barbu, Y. Bréchet, in: J.T. Busby, G. Ilevbare, P.L Andresen (Eds.), 15th International Conference on Environmental Degradation, John Wiley \& Sons, Inc., 2011, p. 1371.

[15] T. Jourdan, J. Nucl. Mater. 467 (2015) 286.

[16] J. Marian, V.V. Bulatov, J. Nucl. Mater. 415 (2011) 84.

[17] M. Gherardi, T. Jourdan, S. Le Bourdiec, G. Bencteux, Comput. Phys. Commun. 183 (2012) 1966.

[18] E. Clouet, A. Barbu, L. Lae, G. Martin, Acta Mater. 53 (2005) 2313, https://doi. org/10.1016/j.actamat.2005.01.038.

[19] T. Jourdan, F. Soisson, E. Clouet, A. Barbu, Acta Mater. 58 (2010) 3400.

[20] T. Jourdan, J.-P. Crocombette, J. Nucl. Mater. 418 (2011) 98.

[21] A.B. Sivak, V.M. Chernov, V.A. Romanov, P.A. Sivak, J. Nucl. Mater. 417 (2011) 1067.

[22] D. Carpentier, T. Jourdan, Y. Le Bouar, M.-C. Marinica, Acta Mater. 136 (2017) 323.

[23] F. Berthier, I. Braems, E. Maras, J. Creuze, B. Legrand, Acta Mater. 58 (2010) 2387.

[24] J. Rottler, D.J. Srolovitz, R. Car, Phys. Rev. B 71 (2005) 064109.

[25] R.E. Stoller, S.I. Golubov, C. Domain, C.S. Becquart, J. Nucl. Mater. 382 (2008) 77.

[26] D. Xu, B.D. Wirth, M. Li, M.A. Kirk, Appl. Phys. Lett. 101 (2012) 101905.

[27] A. Barbu, C.S. Becquart, J.-L. Bocquet, J. Dalla Torre, C. Domain, Philos. Mag. 85 (2005) 541.

[28] J. Dalla Torre, C.-C. Fu, F. Willaime, A. Barbu, J.-L. Bocquet, J. Nucl. Mater. 352 (2006) 42.

[29] C. Ortiz, M. Caturla, J. Comput-Aid. Mater. Des. 14 (2007) 171-181.

[30] D.G. Doran, Rad. Eff. 2 (1970) 249.

[31] H.L. Heinisch, J. Nucl. Mater. 117 (1983) 46.

[32] T. Muroga, Radiat. Eff. Defects Solids 113 (1990) 119.

[33] H.L. Heinisch, Simulating the Production of Free Defects in Irradiated Materials, Technical Report PNL-SA-24441, Pacific Northwest Laboratory, 1994.

[34] H.L. Heinisch, B.N. Singh, J. Nucl. Mater. 232 (1996) 206.

[35] G. Adjanor, S. Bugat, C. Domain, A. Barbu, J. Nucl. Mater. 406 (2010) 175.

[36] C.S. Becquart, C. Domain, Metall. Mater. Trans. A 42A (2011) 852.

[37] T. Jourdan, J.-P. Crocombette, Phys. Rev. B 86 (2012) 054113.

[38] A.Y. Dunn, L. Capolungo, E. Martinez, M. Cherkaoui, J. Nucl. Mater. 443 (2013) 128.

[39] C.J. Ortiz, P. Pichler, T. Fühner, F. Cristiano, B. Colombeau, N.E.B. Cowern, A. Claverie, J. Appl. Phys. 96 (2004) 4866.

[40] C. Borschel, C. Ronning, Nucl. Instr. Meth. B 269 (2011) 2133.

[41] J.F. Ziegler, M.D. Ziegler, J.P. Biersack, Nucl. Instr. Meth. B 268 (2010) 1818.

[42] M.T. Robinson, Phys. Rev. B 40 (1989) 10717.

[43] A.F. Calder, D.J. Bacon, A.V. Barashev, Y.N. Osetsky, Philos. Mag. 90 (2010) 863.

[44] D.J. Bacon, T. Diaz de la Rubia, J. Nucl. Mater. 216 (1994) 275.

[45] Annual Book of ASTM Standards, volume 12.02, ASTM International, 2004

[46] J. Dural, J. Ardonceau, J.C. Jousset, J. Phys. 38 (1977) 1007.

[47] E. Meslin, Mécanismes de fragilisation sous irradiation aux neutrons d'alliages modèles ferritiques et d'un acier de cuve: amas de défauts, Ph.D. thesis, Université de Rouen, 2007.

[48] T. Jourdan, J.-L. Bocquet, F. Soisson, Acta Mater. 58 (2010) 3295.

[49] C.-C. Fu, J. Dalla Torre, F. Willaime, J.-L. Bocquet, A. Barbu, Nat. Mater. 4 (2005) 68.

[50] F. Gao, D.J. Bacon, A.F. Calder, P.E.J. Flewitt, T.A. Lewis, J. Nucl. Mater. 230 (1996) 47.

[51] C. Björkas, K. Nordlund, M.J. Caturla, Phys. Rev. B 85 (2012) 024105.

[52] H.L. Heinisch, J. Nucl. Mater. 103\&104 (1981) 1325.

[53] L. Lunéville, D. Simeone, W.J. Weber, J. Nucl. Mater. 415 (2011) 55.

[54] R. Stoller, G. Odette, B. Wirth, J. Nucl. Mater. 251 (1997) 49-60.

[55] R.E. Stoller, in: R.J. Konings (Ed.), Comprehensive Nuclear Materials, Elsevier, Oxford, 2012, pp. 293-332.

[56] L.E. Rehn, J. Nucl. Mater. 174 (1990) 144.

[57] B. Michaut, T. Jourdan, J. Malaplate, A. Renault-Laborne, F. Sefta, B. Décamps, J. Nucl. Mater. 496 (2017) 166.

[58] S.J. Zinkle, B.N. Singh, J. Nucl. Mater. 199 (1993) 173.

[59] V. Naundorf, M.-P. Macht, H. Wollenberger, J. Nucl. Mater. 186 (1992) 227.

[60] T. Muroga, K. Mihara, H. Watanabe, N. Yoshida, J. Nucl. Mater. 212-215 (1994) 203.

[61] H. Xu, Y.N. Osetsky, R.E. Stoller, J. Nucl. Mater. 423 (2012) 102. 